\title{
NCCLOUD: RECONSTRUCTING DATA USING NUMBER OF CLUSTER-CLOUDS BASED ON NETWORK CODING
}

\begin{tabular}{|l|l|}
\hline Kavyasri N & Mrs. Lokanna Kadakolmath \\
Kavyashr.swamy@gmail.com & Lokanna_vkit@yahoo.co.in \\
Department of computer science and & Department of computer science and \\
Engineering,VKIT, Gudimavu, Bangalore & Enginnering, VKIT, Gudimavu, Bangalore \\
\hline
\end{tabular}

\begin{abstract}
Recent studies suggest to stripe data across multiple cloud vendors to provide fault tolerance for cloud storage. However to preserve data redundancy we need to repair the lost data With the help of other surviving clouds, if a cloud suffers from a permanent failure and loses all its data. The NCCloud is presented, which achieves cost-effective repair for a permanent single-cloud failure, and it is a proxy-based storage system for fault-tolerant multiple-cloud storage. The functional minimum-storage regenerating (FMSR) codes, which is a network-coding-based storage scheme on top of which the NCCloud is built on. FMSR codes uses less repair traffic while maintain the same fault tolerance and data redundancy as in traditional erasure codes (e.g., RAID-6), and, hence, incur less monetary cost due to data transfer. The key design feature of FMSR codes is that during repair we relax the encoding requirement of storage nodes. FMSR codes provide significant monetary cost savings in repair over RAID-6 codes.
\end{abstract}

Index Terms-Regenerating codes, Network coding, Recovery, Implementation.

\section{INTRODUCTION}

At the present world of networking system, for both the developers and the users Cloud computing [2] is one the most important and developing concept. cloud computing is a preferable platform for the persons who are interrelated with the networking environment. In recent days, therefore, protection of data has become a major challenging issue in cloud computing.

Currently, more and more data (such as large scale files, video data and sensory data) need to be stored. So, how to effectively manage these data becomes a major challenge. The distributed storage systems [3] (DSS) are generally employed in practical applications, in order to protect the data from permanent failures of storage servers. In DSS, the files are stored in different servers, so the original files can be recovered from the surviving servers, when a small part of servers fails. The most common method for DSS is replication. In a replicationbased DSS method, reliability can be enhanced, by having multiple copies of an original file. However more data redundancy is introduced by replicating the data. Another useful technology for DSS is Erasure coding [4] [5]. It divides the original file into $N$ encoded sub-files, out of these $N$ sub-files, any $\mathrm{K}$ sub-files can recover the original file, and the nodes can tolerate N-K failures of the storage nodes. Therefore, without sacrificing the reliability, erasure coding can significantly reduce the redundancy. Hence, erasure coding is widely employed in practical applications. However, erasure coding has its disadvantage. The failed sub-file needs to be repaired at a new storage server, when a storage node fails, so that the level of reliability can be maintained. To this end, atleast $K$ different subfiles needs to be downloaded by the client, recover the original file, calculate a regenerating code, and then with the original file and the regenerating code, regenerate a new sub-file. Therefore, how to reduce the required repair bandwidth becomes an interesting topic [6]. Recently, network coding, proposed by Ahlswede et al. [7], is considered as a promising technology for distributed storage systems. A significant feature of network coding is that at intermediate nodes, re-encoding operations are introduced. 
The performance (such as throughput and energy efficiency) of multicast networks is improved from the benefit of re-encoding operations.

In conventional erasure, while striping the data, codes performs well when some clouds experience short-term transient failures or foreseeable permanent failures [8], there are reallife cases showing that permanent failures are not always foreseeable [9], but do occur. From this point of view, my work focuses on unexpected permanent cloud failures. To maintain data redundancy and fault tolerance, it is necessary to activate repair when a cloud fails permanently. A repair operation reconstructs the lost data in a new cloud, by retrieving the data from existing surviving clouds over the network. Moving an enormous amount of data across clouds can introduce significant monetary costs because today's cloud storage providers charge users for outbound data also. Due to data migration, therefore, it is important to reduce the repair traffic (i.e., the amount of data being transferred over the network during repair), and hence, the monetary cost.

To minimize repair traffic, regenerating codes, have been proposed for storing data redundantly in a distributed storage system (a collection of interconnected storage nodes). Most of the organization store their large amount of data in cloud in order to avoid overhead of data managing. To protect the data stored in cloud from corruptions requires fault tolerance to cloud with integrity checking and recovery procedure becomes critical. Failure recovery by using erasure coding requires complex repair traffic. In order to overcome from complex traffic we design regenerating code technique by striping data across multiple servers.

Network coding is a method of optimizing the flow of digital data in a network by transmitting digital evidence about messages. A composite of two or more messages is nothing but "digital evidence". Rather than directly reassembled, the transmitted message is deduced, when the bits of digital evidence arrive at the destination.

In network coding, routers and switches are replaced by devices called coders. The coders transmit metadata in the form of digital evidence about the message along multiple paths simultaneously, rather than directing the packets towards their ultimate destination like blood cells through a system of arteries. Conversely, the metadata which is arriving from two or more sources may be combined in to a single packet. Increasing the effective capacity of a network by minimizing the number and severity of bottleneck is obtained by distribution method. When the network traffic volume is near the maximum capacity obtainable with traditional routing, the improvement is most pronounced. When a destination has enough digital evidence, it can calculate the intended message/packet. Still the original message gets through if the received digital evidence is sufficient, even if some packets on some of the routes are lost or damaged.

We need to repair the corrupted data by replacing original data, if we detect dishonest/corruption in outsourced data. However storing all the data in single server leads to single point of failure. The solution is to stripe data across multiple servers. Thus the failed server can be repaired by a) reading data from other exhisting servers. b) reconstruct the failed server's corrupted data to a new server. To minimize repair traffic regenerating codes have been proposed. On each file, HAIL constructs the protection data and distributes protection data across different servers.

\section{MOTIVATION}

Consider a distributed, multiple-cloud storage setting from a client's point of view, and stripe data over multiple cloud providers. Here I would 
ELK

Asia Pacific Journals

like to propose a proxy-based design, as shown in Fig. 1a, that interconnects multiple cloud repositories. Between client applications and the clouds, this proxy server acts as an interface. The proxy activates the repair operation, if a cloud experiences a permanent failure, as shown in Fig. 1b. That is, the proxy reconstructs new data pieces, on reading the essential data pieces from other surviving clouds, and writes these new pieces to a new cloud.

Given two positive integers $\mathrm{k}$ and $\mathrm{n}$ where $\mathrm{n}>\mathrm{k}$, for reliability an $(\mathrm{n}, \mathrm{k})$ maximum distance separable (MDS) code can be used,

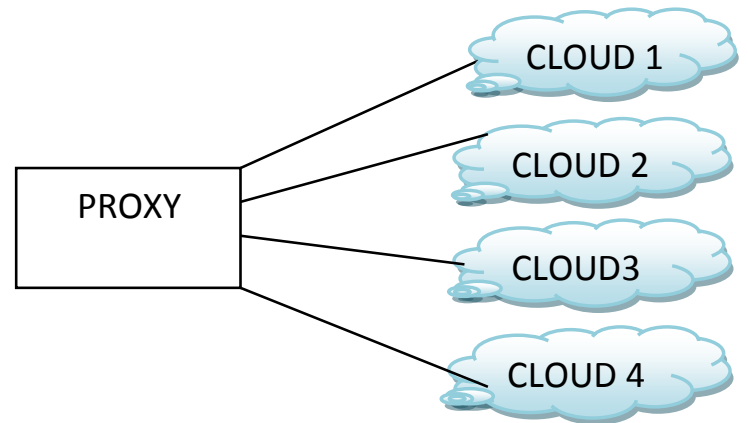

Fig.1.a. Normal Operation

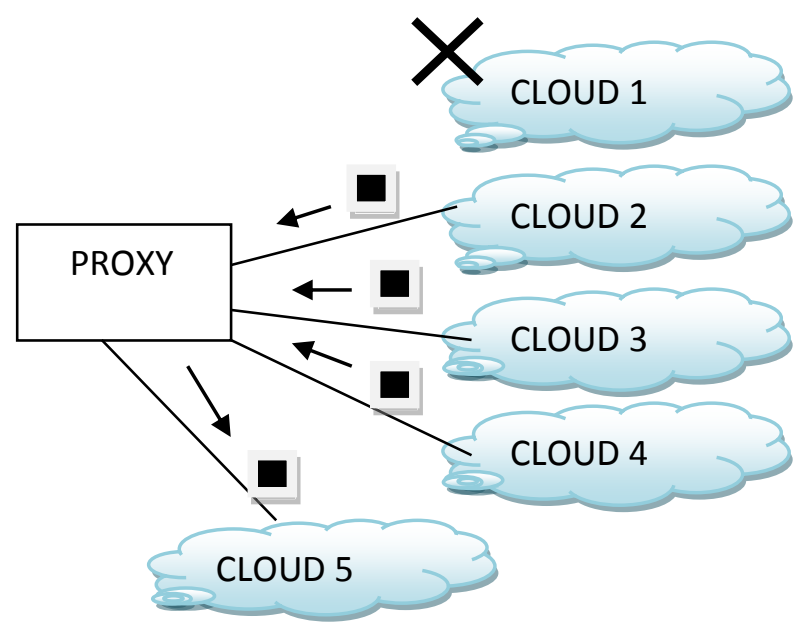

Fig.1.b. Repair operation

initially the data to be stored is divided into $\mathrm{K}$ information packets. Subsequently, to recover the original data (see Fig. 1 for an example), using the MDS code, these are encoded into $\mathrm{n}$ packets (of the same size) such that any $k$ out of these $n$ suffice. In terms of the redundancy-reliability tradeoff, MDS codes are optimal, because $\mathrm{k}$ packets are required to recover the original data that contains the minimum amount of information. In a distributed storage system, at different storage systems (e.g., disks, servers, or peers) the $\mathrm{n}$ encoded packets are stored, spread over a network, and any (n-k) node failures the system can tolerate without data loss. Here I focus on a new problem that arises when storage nodes are distributed and connected in a network. When a storage node of the system fails, the issue of blocks is stored using $(4,2)$ evenod

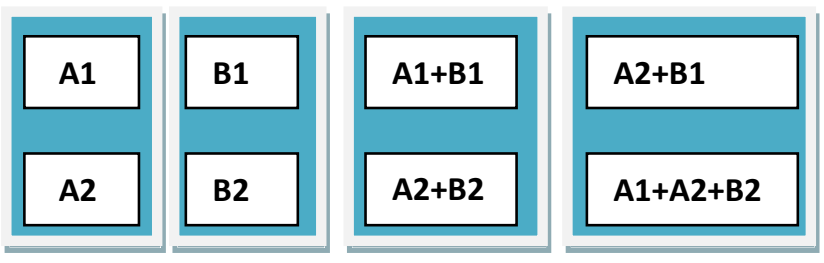

Fig.2. A (4, 2) MDS binary erasure codes

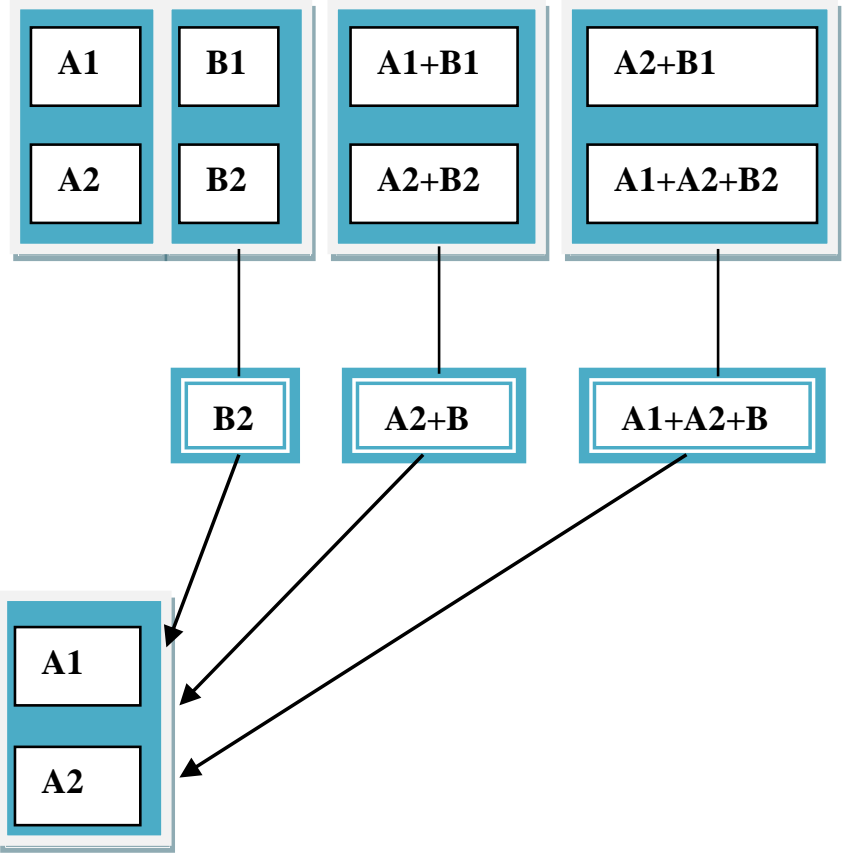

Fig.3. Example of Repair 
code of the previous example and the first node fails. A new node (to be called the newcomer) needs to construct and store two new blocks so that the three existing nodes combined with the newcomer still form a $(4,2)$ MDS code. We call this the repair problem and focus on the required repair bandwidth. Clearly, by assumption any two nodes contain enough information to recover all the data, and hence repairing a single failure is easier than reconstructing all the data, then the newcomer could download four blocks (from any two surviving nodes), reconstruct all four blocks, and store A1, A2. However, it is possible to repair the failure by communicating only three blocks $\mathrm{B} 2, \mathrm{~A} 2+\mathrm{B} 2, \mathrm{~A} 1+\mathrm{A} 2+\mathrm{B} 2$, which can be used to solve for A1, A2 as the example shows.

\section{NCCLOUD DESIGN AND IMPLEMENTATION}

NCCloud is implemented as a proxy that bridges user applications and multiple clouds. Its design is built on three layers. The file system layer presents NCCloud as a mounted drive, which can, thus, be easily interfaced with general user applications. The coding layer deals with the encoding and decoding functions. The storage layer deals with read/ write requests with different clouds. Here it presents the details for implementing FMSR codes in multiple-cloud storage. For FMSR codes on a particular file object which specify three operations: 1) File upload, 2) File download, and 3) Repair. Each cloud repository is viewed as a logical storage node. A thin-cloud interface, is assumed in implementation, such that the storage nodes (i.e., cloud repositories) only need to support basic $\mathrm{read} /$ write operations. Thus, with today's cloud storage services, the FMSR code implementation is compatible. Main property of FMSR codes is that rather than exactly reconstructing the lost chunks, in each repair, we regenerate code chunks that are not necessarily identical to those originally stored in the failed node, as long as the MDS property holds.

Client writes the data to proxy server and proxy server stripes data across multiple servers as shown in the fig.4. A proxy server downloads the corrupted data and reconstructs the corrupted data in new server when any data corruption in the server takes place. Main goal is to reconstruct the lost data of failed server in new server, if one server fails. For a single server failure, the

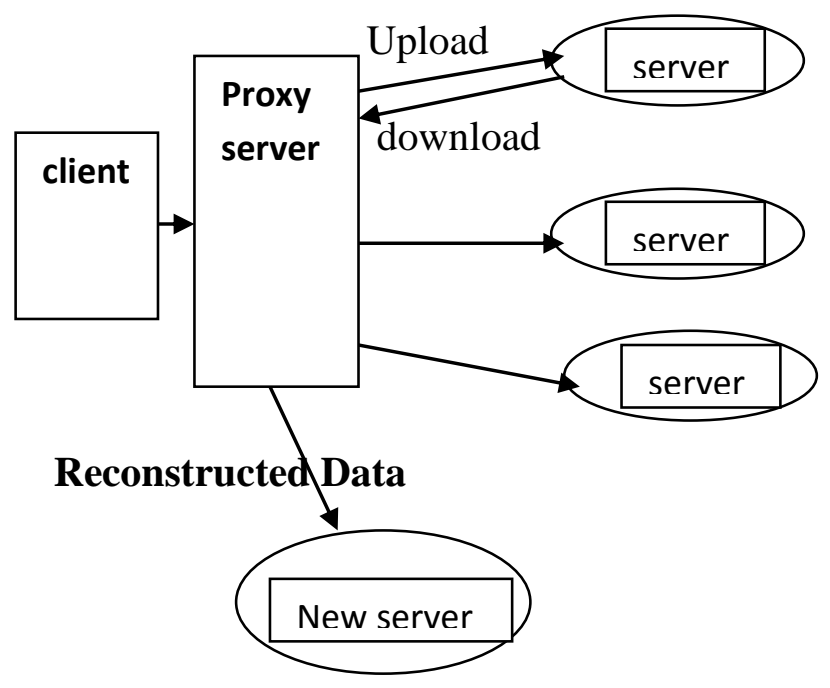

Fig.4. Upload, Download and Repair operations

idea behind repair method is to reconstruct the whole file by contacting other servers.

\section{A. FILE UPLOAD}

Step1: Generate the per file secrets: Generate secret key for each file, Before uploading the file. Without knowing to server, all secret keys are securely stored under client side. We derive each secrete key into multiple keys using derivation function to reduce key management overhead. Using master key encrypt all keys and send into cloud with encrypted key. 
Step2: Using FMSR codes encode the file: To generate code chunk, NCCloud encodes file using the FMSR code.

Step3: Encode each code chunk to generate parity bytes and compute MAC for the first bytes. The parity bytes are downloaded only when error correction is needed.

Step4: Update the metadata file and upload: Upload the code chunks to their respective servers. Attach MAC to all chunks to the metadata.

\section{B. FILE DOWNLOAD}

A file $\mathrm{F}$ from the servers will be downloading as follows

Step1: verify the metadata file.

Step2: For file F, download and decode FMSR code chunks. We download $\mathrm{k}(\mathrm{n}-\mathrm{k})$ chunks from any $\mathrm{k}$ servers to construct file $\mathrm{F}$. After downloading a code chunk, we verify MAC corresponding to it.

\section{REPAIR OPERATION}

Repair operation is used when server fails. Repair operation include

Check the metadata file.

Download and decode the needed chunks.

$\square$ Encode, update metadata and upload

\section{RESULT}

The monthly price plans for three major providers as of May 2013 as shown in Table 1. From the first chargeable usage tier, the cost is taken. Now during storage repair, can save 25-50 percent of the download traffic. The number of chunks and the storage size being generated per file object are the same in both RAID-6 and FMSR codes (assuming that we aggregate chunks in FMSR codes).Fig. 8 plots the response times of repair operation(with 95 percent confidence intervals plotted) versus the file size. FMSR codes have slightly less response time in repair. The main advantage of FMSR codes is that during repair, FMSR codes download less data. Under this cost model, the monthly storage cost drops to only $\$ 13,107$ for both RAID-6 and FMSR codes. However, the repair cost for RAID-6 codes is $\$ 66,662$, while that for FMSR codes is $\$ 39,137$, with a saving of $\$ 27,525$.

\begin{tabular}{|l|l|l|l|}
\hline & S3 & RS & AZURE \\
\hline $\begin{array}{l}\text { Storage (per GB) } \\
\text { Data transfer in(per GB) } \\
\text { Data transfer out(per }\end{array}$ & Free & Free & Free \\
$\begin{array}{l}\text { GB) } \\
\text { POST, PUT(per 10K } \\
\text { request) } \\
\text { GET(per 10K request) }\end{array}$ & $\$ .12$ & $\$ .12$ & $\$ .12$ \\
\hline
\end{tabular}

Table. 1 Monthly Price Plans (in US Dollars) for Amazon S3 (US Standard), Rackspace Cloud Files and Windows Azure Storage (North America and Europe), as of May 2013

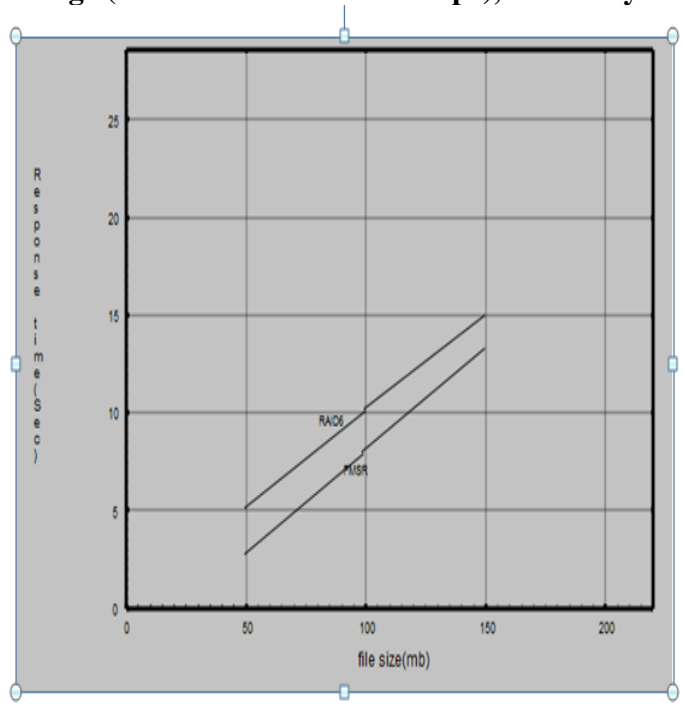

Fig. 5 Response time of repair operation 
ELK

Asia Pacific Journals

\section{CONCLUSIONS \& FUTURE WORK}

NCCloud, a proxy-based multiple-cloud storage system is presented that practically addresses the reliability of today's cloud backup storage. NCCloud not only provides fault tolerance in storage, but also allows cost-effective repair when a cloud permanently fails. NCCloud implements a practical version of the FMSR codes, which regenerates new parity chunks during repair subject to the required degree of data redundancy. FMSR code implementation eliminates the encoding requirement of storage nodes (or cloud) during repair, while ensuring that the new set of stored chunks after each round of repair preserves the required fault tolerance. The correctness of double-fault tolerance is still to be proven. In future work, we also plan to conduct further reliability analysis using more effective metrics

\section{REFERENCES}

[1] Henry C.H. Chen, Yuchong Hu, Patrick P.C. Lee, And Yang Tang, "Nccloud: A NetworkCoding-Based Storage System In A Cloud-OfClouds"

[2] Yashpal Kadam, "Security Issues in Cloud Computing A Transparent View", International Journal of Computer Science Emerging Technology,

[3] Chang F, Dean J, Ghemawat S, Hsieh WC, Wallach DA, Burrows M, Chandra T, Fikes A, Gruber RE. Bigtable: A distributed storage system for structured data.

[4] L Rizzo, Effective erasure codes for reliable computer communication protocols.

[5] L Xu, J Bruck. X-code: MDS array codes with optimal encoding.

[6] KV Rashmi, NB Shah, PV Kumar, K Ramchandran. Explicit construction of optimal exact regenerating codes for distributed storage.
ELK Asia Pacific Journals - Special Issue ISBN: 978-81-930411-5-4

[7] Ahlswede R, Cai N. Network Information Flow.

[8] H. Abu-Libdeh, L. Princehouse, and $\mathrm{H}$. Weatherspoon, "RACS: A Case for Cloud Storage Diversity,"

[9] H. Blodget, "Amazon's Cloud Crash Disaster Permanently Destroyed Many Customers' Data," 